NOTICIAS Y COMENTARIOS 


\title{
EL MODELO TURÍSTICO DE LA MARINA ALTA: APUNTES PARA UNA NUEVA ETAPA DESDE LA SOSTENIBILIDAD COMO REFERENCIA*
}

\author{
J. Fernando Vera Rebollo \\ Instituto Universitario de Geografía
}

\section{RESUMEN}

El propósito de las siguientes reflexiones no es otro que aportar algunas ideas en el marco de un debate necesario sobre el modelo territorial-turístico de la Marina Alta, en el contexto de una nueva filosofía de desarrollo que lleva implícitos, como contenidos clave, la gestión racional de los recursos naturales y culturales y la calidad de vida de las poblaciones locales. Son aspectos a los que el turismo puede aportar argumentos, en tanto que su futuro como actividad ha de estar basado en la calidad, la gestión ambiental y la diferenciación de los espacios de destino.

Palabras clave: desarrollo turístico, sostenibilidad, gestión de recursos naturales y culturales, ordenación supramunicipal, espacio comarcal.

\begin{abstract}
The aim of the following reflections is to provide some ideas about the territorialtourist model of the Marina Alta (Valencian Region) in the framework of a necessary debate and in the context of a new development philosophy distinguished by key issues as the rational management of natural and cultural resources and quality of life for local communities. Tourism can contribute to these goals because its future must be based on quality, environmental management and differentiation of destination areas.
\end{abstract}

Key words: tourist development, sustainability, cultural and natural resources management, supramunicipal land-use planning, subregional areas.

* Síntesis de la ponencia presentada en las Jornadas organizadas por el Institut d'Estudis Comarcals de la Marina Alta, Teulada, Mayo de 1999. 


\section{La apuesta necesaria hacia una fase basada en la calidad ambiental y en la gestión de los recursos naturales y culturales}

Es evidente que el actual sistema de uso turístico de la comarca, aunque muy polarizado en la franja litoral y en algún municipio prelitoral, es el principal factor en la reorganización del espacio económico y en sus dinámicas de transformación. Por tanto, entre los retos para construir el propio futuro es esencial, entre otros aspectos, racionalizar los procesos de implantación, coordinar de forma eficiente el aprovechamiento de los recursos, gestionar el territorio desde la perspectiva del largo plazo, apostar por un sistema de oferta cualificada y, sobre todo, no confundir el desarrollo turístico con un objetivo de crecimiento continuado e indiferenciado de la oferta.

El nivel de especialización alcanzado en la actividad turística va estrechamente unido a la creación de nuevos asentamientos que configuran un sistema de marcado carácter residencial, asociado al consumo de suelo rústico y a una pérdida progresiva e incesante del papel que tradicionalmente ha tenido la actividad agraria y de sus paisajes y formas de organización del territorio. La conformación de una metrópolis rural o campo urbanizado, según lo definía el arquitecto A. Peñín, diferencia a la Marina Alta de otros modelos próximos. Pero la diferencia no es sólo morfológica, ya que el tirón de la demanda, desde el decenio de los sesenta, justifica la importancia adquirida por el subsector de la construcción y afines, clave en la creación de empleo y renta en estos municipios. Y como consecuencia de la magnitud del volumen de oferta generada, se ha ido consolidando un aparato de servicios que, junto con la construcción, definen los rasgos funcionales de un sistema de poblamiento disperso, nuevos espacios urbanizados para las demandas de ocio, que han reorganizado el sistema económico-territorial de la comarca.

Hoy por hoy la incuestionable especialización en este singular modelo de urbanismo de ocio obliga a repensar las claves del futuro, desde el propio marco territorial que constituye la comarca, a la hora de sentar principios de coordinación funcional (infraestructuras, equipamientos, servicios públicos, asignación de recursos de agua) y de congruencia en la gestión ambiental (espacios naturales, paisajes rurales, vertidos y depuración) y territorial (clasificación de suelo en los planeamientos urbanísticos municipales). Todo ello sin olvidar que, frente a la competencia y descoordinación, una estrategia turística de futuro pasa necesariamente por la forma de rentabilizar los beneficios de la complementariedad entre actividades y territorios vecinos.

El reconocimiento de las singularidades de este modelo de implantación, como nivel de análisis interno, ha de llevarnos a entender que seguir viviendo del turismo y conseguir una posición competitiva, en el escenario de espacios y áreas receptoras, exige el tránsito progresivo, pero decidido, hacia formas y parámetros más cualitativos en la creación de la oferta, como contenido del sistema turístico. Mientras que el continente tiene en la calidad del paisaje su principal argumento de diferenciación. La revalorización de espacios que mantienen calidad ambiental y paisajística, la conservación del patrimonio cultural y etnográfico y la propia concienciación de las sociedades locales sobre la gestión de los recursos y su relación sinérgica con la calidad del turismo, son vías de trabajo para esta nueva etapa.

Desde el análisis externo, la difusión de nuevas tendencias motivacionales entre los consumidores turísticos, se perfila como factor de oportunidad, siempre que se actúe a tiempo para afianzar ventajas competitivas; a la vez que riesgo, si se mantienen las inercias. Se trata pues de sentar bases de futuro para una actividad cuyo crecimiento futuro será más complejo y diversificado y no podrá basarse en la reducción de precios y consiguiente desgaste de la calidad de instalaciones y entornos. 
A estas alturas no sirve la permisividad y el dejar hacer en los procesos de implantación, sino que es preciso gestionar y concertar con los agentes sociales y empresariales las líneas del propio futuro del turismo. De igual modo, tampoco tiene sentido negar el papel que hoy tiene el turismo en este espacio, en tanto que sería desconocer la realidad. Una realidad que reclama cualificación y en la que se presentan no pocas contradicciones; desde los discursos y recomendaciones globales que apuntan hacia la sostenibilidad, hasta las inercias basadas en la instrumentación inmobiliaria del turismo, sin olvidar los esfuerzos puntuales de agentes comprometidos y la misma dificultad para poner en marcha técnicas y procedimientos de gestión. Además, por su propio contenido, las estrategias de desarrollo turístico que pretendan ser exitosas requieren un marcado sentido integrador entre diferentes políticas (medio ambiente, urbanismo, cultura y patrimonio, infraestructuras, agricultura, entre otras) y agentes económicos actuantes (hoteleros, inmobiliarios, promotores, comerciantes, etc).

\section{Debilidades y fortalezas del modelo turístico comarcal}

Entre lo que puede denominarse debilidades o puntos débiles del sistema de turístico, se apuntan las siguientes:

- Como hecho general, una de las consecuencias más claras derivadas del modelo de implantación del turismo en la estructura comarcal es el desequilibrio y el consiguiente dualismo entre la franja costera, espacio donde se polarizan las inversiones e iniciativas y se concentra la población, además de acelerarse las transformaciones territoriales y ambientales. Frente al litoral congestionado por nuevos usos y funciones, aparece un interior rural en proceso de despoblación y abandono de los usos y actividades tradicionales que gestionaron desde época histórica el territorio y el paisaje y contribuyeron a definir sus señas de identidad. El desequilibrio no sólo se reproduce en población, renta, empleo, etc, sino en parámetros como son los desplazamientos pendulares de mano de obra, o en la concentración de las inversiones públicas. Será preciso pues considerar la capacidad de las nuevas formas de desarrollo del turismo como factor de reequilibrio territorial y de compensación interiorlitoral. Además de las iniciativas empresariales que empiezan a cristalizar en municipios interiores y del apoyo del programa LEADER II, gestionado por el CEDER Aitana, tiene gran interes en el plano territorial y ambiental el desarrollo de propuestas supramunicipales integradoras, en el marco del CONCERCOST, proyecto financiado por la U.E. para el espacio de las Comarcas Centrales Valencianas, entre las que se incluye la Marina Alta.

- El sistema turístico comarcal tiene uno de sus rasgos más representativos y problemáticos en el consumo de suelo por la urbanización, a lo que se añade la desarticulación de los nuevos asentamientos y comunidades, en relación a la estructura del poblamiento tradicional comarcal. A ello se añade, en no pocos casos, la carencia que tales asentamientos padecen en materia de infraestructuras y equipamientos. Será preciso articular racionalmente el nuevo sistema de poblamiento y dotarlo de forma adecuada. Pero, esencialmente se trata de racionalizar las apetencias de crecimiento continuado de estos asentamientos, a través del planeamiento municipal.

- Dentro de las debilidades estructurales del sistema de uso turístico del territorio nos parece especialmente importante hacer frente a la degradación que presentan determinados tramos costeros, para los que será preciso actuar con medidas de protección y restauración de su valor ambiental y paisajístico. 
- Desde el lado de la oferta turística es manifiesto el desequilibrio entre el alojamiento en viviendas y apartamentos, que supone la parte más sustancial de la misma (véanse los cuadros adjuntos), frente a un peso muy reducido de la oferta en hoteles, hostales y campamentos. Es cierto que una parte muy notable de este alojamiento residencial responde al establecimiento de ciudadanos procedentes de otros países, un mercado de gran tradición e importancia en la comarca; al que se añade la segunda residencia de propietarios de otras regiones españolas. Pero también cabe añadir el problema que supone la competencia, por parte de un considerable volumen de viviendas y apartamentos, cuando entran en los circuitos comerciales del alojamiento sin estar declaradas como viviendas de uso turístico. Cabe citar como excepción las empresas que gestionan de forma oficial alquileres y servicios en este tipo de oferta y la aparición de complejos residenciales que, de este modo, contribuyen a dinamizar la ocupación del alojamiento en determinados municipios.

- El abrumador predominio de un producto turístico-residencial indiferenciado resta oportunidades para aprovechar las excelentes condiciones con que cuenta la comarca para orientar las intervenciones hacia productos más especializados, en relación con la segmentación de la demanda. Debe apoyarse un verdadero plan de estructuración de productos específicos, con base en los recuros naturales y culturales y aprovechando el alojamiento existente, en el marco más global del atractivo que suponen el clima y el mar.

- Esta línea entronca con otra de las debilidades, como es la excesiva dependencia del subsector de la construcción y afines. Con ser su papel esencial en el sistema económico comarcal y en su estructura de empleo, conviene diversificar la estructura actual y apoyar la creación de empresas de prestación de servicios, en línea con las acciones que se plantean.

- Aunque no es un problema específico de la Marina Alta y suele presentar como hecho generalizado para las distintas áreas y destinos turísticos, ha fallado la coordinación administrativa en el proceso de consolidación del sistema turístico. Es notable, entre otros aspectos, la descoordinación y desinterés entre las diferentes áreas de gobierno municipal a la hora de dar el tratamiento integrador que el turismo, como función económica con notables implicaciones territoriales, culturales y medioambientales, tiene en los municipios.

En el mismo tema, pesa la descoordinación administrativa en sentido vertical, es decir, entre los Ayuntamientos, la Generalitat y el gobierno central, respecto a cuestiones que, aún desperdigadas en el marco competencial, se perciben y presentan como problemas en el nivel local.

- La apuesta por el desarrollo sostenible junto con el esfuerzo por rentabilizar los espacios naturales protegidos como escenario de prácticas y visitas recreativoeducativas requiere, de una vez por todas, un esfuerzo en la gestión de tales espacios, cuestión que falla de forma clara en la actualidad.

Pero la caracterización del modelo actual no sería rigurosa sin atender a los puntos fuertes que presenta el sistema; aunque algunos de ellos responden más al mantenimiento de las tradicionales ventajas comparativas iniciales que a los esfuerzos por afianzar verdaderas ventajas competitivas, basadas en la calidad y la gestión eficiente de los recursos. Lo cierto es que la permanencia de tales factores es hoy la clave para poder articular nuevas bases de trabajo:

- A pesar de la espectacular transformación del paisaje natural y rural de la comarca impuesta por el proceso de urbanización asociado al turismo, y de forma especial en su franja costera, todavía se mantienen espacios rurales y naturales con extrordina- 


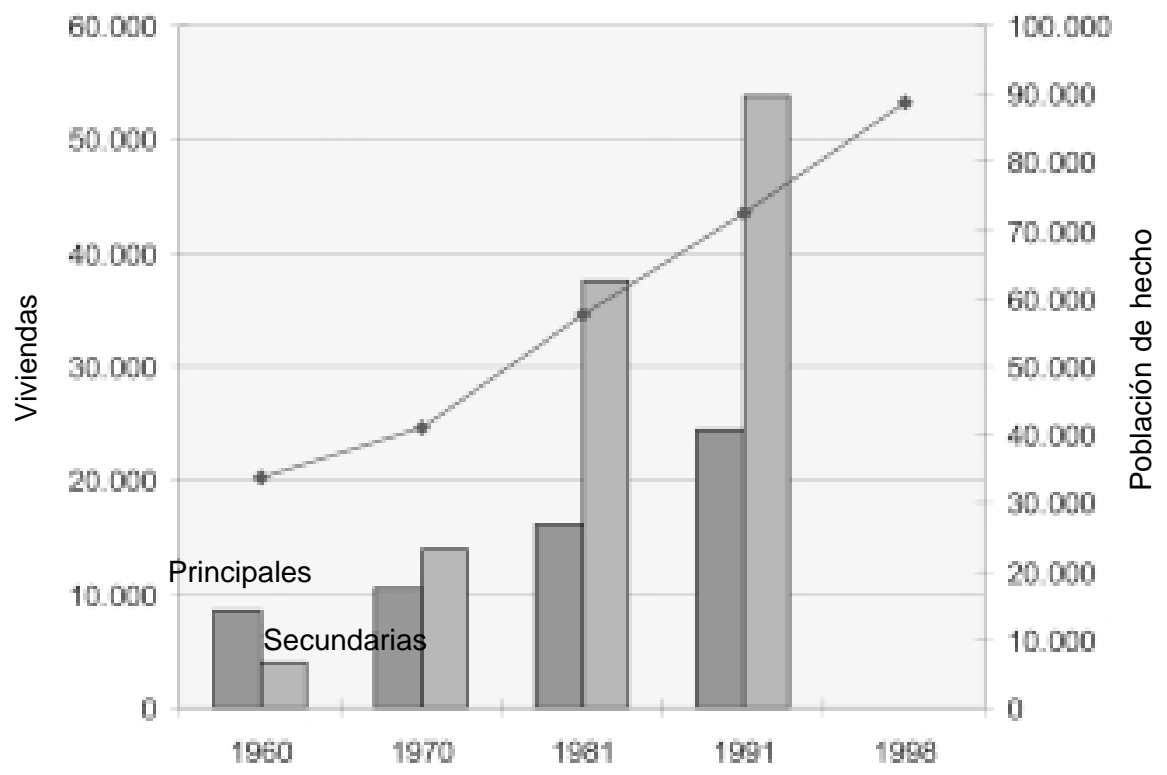

Figura 1. Evolución de la población de hecho y viviendas totales en los municipios de Benidoleig, Benissa, Benitatxell, Calp, Dénia, Teulada, Xábia y El Verger (1960-1998).

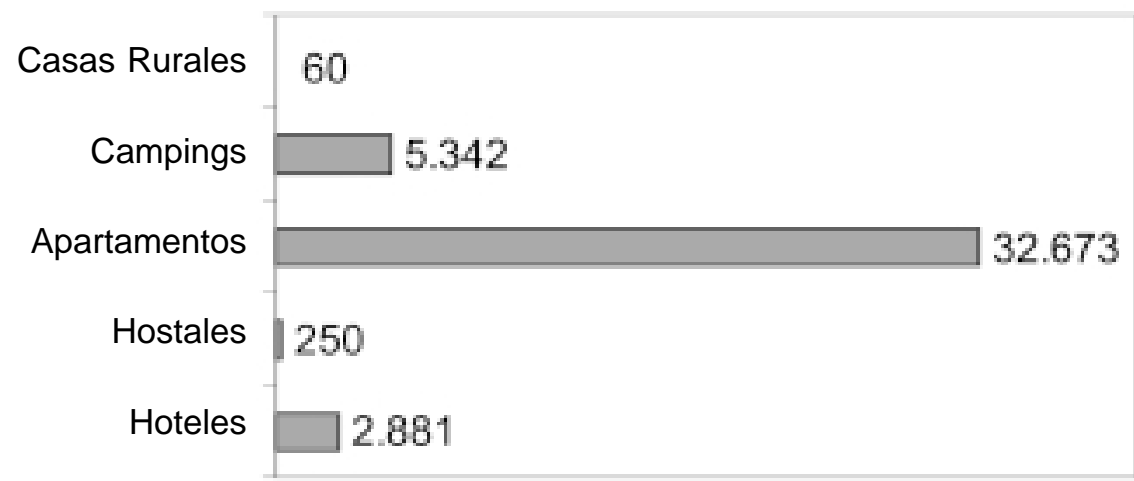

Figura 2. Oferta de alojamiento turístico reglado en la Marina alta, 1998 (númeo de plazas).

rio valor medioambiental y paisajístico. A ellos se añade la pervivencia de núcleos de población que conservan sus identidades y patrimonio edificado, además de las señas de identidad que definen el sustrato cultural. Una política turística basada en la sostenibilidad puede aportar argumentos para la conservación, revalorización y gestión de este patrimonio natural y cultural.

- La Marina Alta ofrece, en el contexto del País Valenciano, una excelente «renta de situación», fruto de la combinación entre condiciones climáticas y accesibilidad desde regiones y áreas emisoras, lo cual constituye un factor de primera magnitud a la hora de diseñar estrategias de posicionamiento en el mercado turístico. Pero la 
cuestión es saber diferenciar el producto, en un contexto de espacios repectores masivos y banalizados.

- La imagen turística comarcal en el exterior se asocia a una mayor calidad ambiental y paisajística, frente a otros espacios del propio País Valenciano que se identifican como lugares más degradados. Debe aprovecharse este factor precisamente para afianzar la línea de calidad que venimos defendiendo. Sobre todo porque es un espacio atractivo para demandas que saben apreciar tales componentes de oferta y entorno.

- La especialización alcanzada en prestación de servicios turísticos dentro de los municipios litorales y en algunos prelitorales permite hoy contar con profesionales y técnicos preparados para gestionar la actividad, además de la misma presencia de empresarios comprometidos con su realidad económica y social que ven la necesidad de afianzar el turismo como clave de futuro, desde presupuestos más equilibrados y sostenibles.

- El actual volumen de plazas de alojamiento justifica una posición en determinados mercados, como el residencial, que puede ser el acicate para el lanzamiento de nuevos productos más dinámicos y desestacionalizadores.

- Este sistema turístico, a pesar de las consecuencias de la espontaneidad y falta de planificación, ha permitido generar un entramado económico, a través de efectos inducidos y del multiplicador de actividad, que beneficia a otros sectores y ramas productivas, hasta tal punto que es un ejemplo de integración de la nueva actividad en la estructura económica y social de las poblaciones de este ámbito. Son razones para apostar por la complementariedad entre actividades en relación con el propio futuro del turismo.

El análisis interno de la situación debe complementarse y conjugarse, a la hora de apuntar algunas claves y líneas de futuro, con el conocimiento del escenario turístico global. De este modo, el hecho más notable es la tendencia al crecimiento de la actividad turística, aunque en un contexto de fuerte competencia entre destinos y espacios receptores y con unas exigencias crecientes por parte de la demanda, en cuanto a calidad y autenticidad de los productos/servicios y de respeto al medio ambiente. Los cambios cualitativos desde el lado de los mercados emisores fuerzan a corregir situaciones y superar inercias y contradicciones del modelo actual, al tiempo que permiten configurar nuevos productos más diversificados y especializados, que no han de suponer un crecimiento continuado del volumen de plazas indiferenciadas sino la revalorización de los recursos endógenos. Entre otros factores, el efecto del fraccionamiento de las vacaciones y la propensión hacia un mayor número de viajes más repartidos a lo largo del año es una oportunidad para dinamizar actividades de ocio y turismo y, por ende, rentabilizar de forma más eficiente la oferta actual y los equipamientos asociados; en definitiva, un marco global que impulsa a centrar los esfuerzos en la gestión de la actividad.

\section{Algunas vías de trabajo}

Se sugieren a continuación algunas vías de trabajo, sin pretensiones de exhaustividad, con el propósito de articular un marco operativo que permita cualificar el modelo turístico actual y sentar nuevos principios y bases de intervención en aras de la sostenibilidad.

a) El medio físico-ecológico de la comarca y su realidad funcional y sociocultural permiten la definición de una estructura territorial general, sobre la que sentar directrices y recomendaciones relativas a localización de actividades y usos del suelo, sistemas generales e infraestructuras, equipamientos y dotaciones comunitarias, distribución de la oferta de alojamiento y complementaria, espacios protegidos 
y conservación del paisaje natural y rural, entre otros contenidos que remiten de forma inequívoca a la elaboración de un plan de acción territorial, de ámbito comarcal que, lejos de ser un documento técnico más, habrá de basarse, para su formulación, en la participación ciudadana para el desarrollo de propuestas, así como para sentar los principios de integración territorial, social, económica y ambiental que deben sustentarlo.

b) En la misma línea, se debe entender la necesidad de coordinar y adecuar los instrumentos de planeamiento municipal a la nueva realidad, desde nuevos referentes de calidad y diferenciación de la oferta que implican ajustar el crecimiento del alojamiento y una apuesta decidida por la gestión del suelo como recurso. Paralelamente es prioritario establecer medidas para la recuperación del patrimonio edificado, especialmente el asociado al medio rural y al poblamiento tradicional, así como la fijación de estándares relativos a densidades, equipamientos, espacios libres de uso público e integración de la arquitectura en el paisaje, para las nuevas actuaciones.

c) El título IV de la Ley de Turismo de la Generalitat Valenciana recoge la idea del Plan Director de los Espacios Turísticos de la Comunidad Valenciana, cuyo documento de bases fue presentado en 1995, pero que nunca llegó a desarrollarse. La idea de deslindar ámbitos de planificación turística sobre la base de complementariedades e intereses comunes, así como el establecimiento de líneas de actuación concertadas en materia de producto, promoción, comercialización y formación turística, nos parece una vía de actuación adecuada para estructurar el futuro del turismo a escala comarcal.

d) Desde el propio ámbito municipal, la política turística, más allá de meras acciones promocionales, debe coordinarse de forma eficiente con urbanismo y medio ambiente para establecer los fundamentos de un modelo basado en productos específicos, capaces de rentabilizar el potencial de recursos locales, con más valor añadido que las ofertas de alojamiento indiferenciado al uso. Acometer líneas de actuación como la restauración de espacios degradados en el litoral, revalorizar los ámbitos con valor ecológico-paisajístico mediante una adecuada gestión, orientar la creación de oferta hacia establecimientos que hagan del tema ambiental y de la calidad su principal atractivo, fomentar el uso y restauración del patrimonio edificado frente a la construcción de nueva planta, entre otras líneas. Para los espacios consolidados por el llamado turismo residencial se trata de reurbanizar y mejorar las dotaciones y servicios, mediante un compromiso público-privado.

\section{A modo de reflexiones finales}

El proceso de desarrollo del sistema turístico comarcal, clave de su actual organización espacial y económica, ha de orientarse en los próximos años hacia el logro de unos objetivos de sostenibilidad, a través de la diferenciación y calidad de sus componentes de oferta y de la gestión del patrimonio natural y cultural que le atribuye su sentido. El logro de una posición competitiva no se basa en la velocidad de los procesos de crecimiento o en las magnitudes de oferta, sino en la capacidad de afianzar una actividad rentable en términos económicos y sociales, capaz de contribuir a la conservación y gestión de los recursos y al reequilibrio territorial del espacio comarcal. Frente al crecimiento indiferenciado y polarizado, las nuevas tendencias motivacionales de la demanda y la propia congruencia en los procesos de implantación de las actividades turísticas y de ocio hacen aconsejable la apuesta por los productos singularizados, la gestión del paisaje, la autentici- 
dad en la experiencia turística, la recuperación del patrimonio edificado y la restauración de los espacios degradados.

Las nuevas claves de futuro requieren una coordinación eficiente en temas como el urbanismo, la gestión del suelo rústico y de los espacios protegidos, el trazado y aprovechamiento de las infraestructuras, la modernización y dinamización de la oferta de alojamiento, la complementariedad entre territorios que comparten áreas funcionales o fisiográficamente homogéneas. De igual modo, el diseño del propio futuro exige un compromiso ciudadano y una concertación público-privada a la hora de sentar nuevos criterios. En definitiva, son líneas que conducen a repensar e insistir en el papel del ámbito comarcal/ local como espacio de referencia y de identidad social ya que el primer y principal objetivo de un modelo de desarrollo es la calidad de vida de las poblaciones locales.

\section{Bibliografía}

AGUILÓ PÉREZ, E. (1996): «Factores de cambio en el turismo: políticas a desarrollar», en Valdés, L.; Vega, A. (coord.), Turismo y promoción de destinos turísticos: implicaciones empresariales. Servicio de Publicaciones de la Universidad de Oviedo, p. 21-40.

ARAUJO, J. (1997): «El Mediterráneo, un mar cansado» en El Mediterráneo desde la otra orilla. Alacant, CAM, p. 157-172.

BOTE GÓMEZ, V. (1995): «Estructura y desarrollo del turismo en España: hacia un cambio cualitativo y más responsable», en Economía del turismo, Colegio de Economistas de Las Palmas de Gran Canaria.

BOTE GÓMEZ, V.; MARCHENA GÓMEZ, M. (1995): «Política turística», en Introducción a la economía del turismo en España. Madrid, Cívitas, p. 295-326.

BRIASSOULIS, H.; VAN der STRAATEN, J. (1992): Tourism and the environment; regional, economic and policy issues. Dordrecht, Kluwe Academic Publishers.

COCCOSSIS, H.; PARPAIRIS, A. (1992): «Tourism and the environment. Some observations on the concept of carrying capacity». Tourism and the environment. Dordrecht, Kluwer, p. 23-32.

COCCOSSIS, H.; NIJKAMP, P. (eds.) (1995): Sustainable tourism development. Hamsphire, Avebury, $198 \mathrm{p}$.

ESTEBAN TALAYA, A. (1996): «Comportamiento de la demanda turística en España. Realidad actual y previsiones», en Valdés y Vega, $O b$. cit, p. 41-58.

GENERALITAT VALENCIANA (1990): Libro blanco del turismo en la Comunidad Valenciana. València, Conselleria d'Indústria, Comerç i Turisme, $394 \mathrm{p}$.

HUNTER, C.; GREEN, H. (1995): Tourism and the environment. A sustainable relationship? Londres, Routledge, $212 \mathrm{p}$.

IOANNIDES, D.; DEBAGGE, K. (1997): «Post-fordism and flexibility: the travel industry polyglot». Tourism Management, 18 (4), p. 229-241.

IRISO, A. (1997): «El turismo en áreas protegidas», en La actividad turística española en 1996. Edición 1997, Madrid, AECIT; Nexo edit., p. 451-472.

LANQUAR, R. (1995): Tourisme et environnement en Mediterranée. Enjeux et prospective. París, Economica et Plan Bleu, $174 \mathrm{p}$.

MARCHENA, M.; VERA REBOLLO, J.F. (1995): «Coastal areas: processes, typologies ands prospects», en A. Montanari i A.M. Williams, European tourism. Regions, spaces and restructuring, Chichester, John Wiley and Sons, p. 111-126.

PEÑÍN, A. (1988): «La Marina, metrópolis rural o campo urbanizado», en Urbanismo-COAM, núm. 4 , monogràfic sobre urbanism en àrees turístiques, Colegio de Arquitectos de Madrid.

PRATS, J.M. (1998): «El proyecto europeo de Carta del Turismo Sostenible en espacios protegidos», en Hacia un turismo sostenible en la reserva de la biosfera de Urdaibai. Dept. d'Ordenació del Territori, l'Habitatge i el Medi Ambient del Govern Basc, p. 189-192.

PRATS PALAZUELO, F. (1995): Turismo, territorio y medio ambiente: el caso del Plan Insular de Lanzarote. Cabildo de Lanzarote, Conferencia Mundial de Turismo Sostenible, vol. 6, 47 p. 
STABLER, M.J. (edit.) (1997): Tourism sustainability. Principles to practice. Oxon, CAB International, $381 \mathrm{p}$.

VERA, J.F.; MARCHENA, M. (1995): «El modelo turístico español», en Introducción a la economía del turismo en España. Madrid, Cívitas, p. 327-364.

VERA REBOLLO, J.F.; RIPPIN, R. (1996): «Decline of a Mediterranean tourist area and restructuring strategies: the Valencian Region», en Priestley, Edwards \& Coccossis (edit.), Sustainable Tourism? European experiences. Oxon, CAB International, p. 120-136.

VERA REBOLLO, J.F. (1999): «Los usos humanos del litoral: conflictos y complementariedades», en La dinámica litoral-interior, Universidade de Santiago de Compostela; Asociación de Geógrafos Españoles.

VERA, J.F.; LÓPEZ PALOMEQUE, F.; MARCHENA, M.; ANTON, S. (1997): Análisis territorial del turismo: una nueva geografía del turismo. Barcelona, Ariel, $443 \mathrm{p}$.

WTO (1997): Tourism market trends. Europe, edition 1997. Madrid, World Tourism Organization, $174 \mathrm{p}$. 
